\title{
Chemcomm
}

Chemical Communications

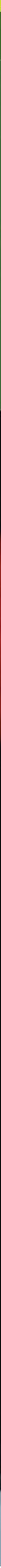

ISSN 1359-7345

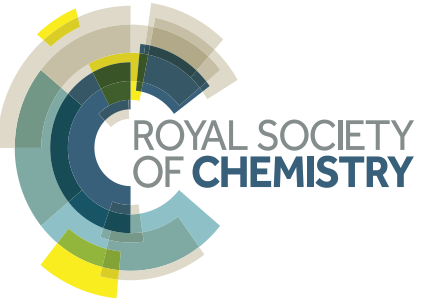


Cite this: Chem. Commun., 2015,

51, 5590

Received 14th February 2014,

Accepted 6th May 2014

DOI: $10.1039 / c 4 c c 01190 b$

www.rsc.org/chemcomm

\section{Ultrafast on-site selective visual detection of TNT at sub-ppt level using fluorescent gold cluster incorporated single nanofiber $\dagger$}

\author{
Anitha Senthamizhan, ${ }^{\star a}$ Asli Celebioglu ${ }^{a b}$ and Tamer Uyar ${ }^{a b}$
}

In this communication, a fluorescent gold cluster incorporated electrospun nanofibrous membrane and single nanofiber for selective and sensitive detection of TNT at sub-ppt level are demonstrated.

The development of an instant on-site visual detection system for trinitrotoluene (TNT) is a critical need for a secured society and a greener environment. ${ }^{1}$ Despite momentous advances in the field, a portable and reliable method for quick and selective detection of TNT still poses a challenge to many, for reasons including inappropriate usage in subordinate areas and untrained personnel. ${ }^{2}$ Recently, efforts have been directed towards fluorescence quenching based sensors because of their acute sensitivity and simple on/off detection. As of now, fluorophores, conjugated polymers, and fluorescent quantum dots have been used as reporter materials., In spite of the enormous attempts being made, the necessity of tagging makes it expensive to use these materials as sensors in real life applications. Also, appropriate exploration of fluorescent metal cluster-based sensors remains at a premature stage. The main reason underlying this fact is the lack of durability under demanding conditions, cost, etc., all of which are necessary for perception of the day-to-day life requirements for on-the-spot detection of TNT. Here, we are interested in filling this gap and studying the possibility of preparing a fluorescent nanofibrous hybrid system with superior selectivity and detection of TNT at sub-ppt level.

We demonstrate in this article for the first time the selective, on-the-spot detection of TNT at sub-ppt level using a single nanofiber embedded fluorescent gold cluster hybrid system. In this effort, the gold clusters are incorporated in the nanofiber (NF) using a simple and straightforward electrospinning method. Polyethylene oxide (PEO) was selected as the matrix owing to its excellent electrospinnability. The most challenging

\footnotetext{
${ }^{a}$ UNAM-National Nanotechnology Research Center, Bilkent University, Ankara, 06800, Turkey.E-mail: senthamizhan@unam.bilkent.edu.tr, uyar@unam.bilkent.edu.tr

${ }^{b}$ Institute of Materials Science \& Nanotechnology, Bilkent University, Ankara, 06800, Turkey

$\dagger$ Electronic supplementary information (ESI) available: Experimental procedure, SEM images, PL spectra and additional figures. See DOI: 10.1039/c4cc01190b
}

step is the homogeneous distribution of clusters in NFs with the preservation of their optical properties. ${ }^{5,6}$ Precise optimization and many efforts have led to the accomplishment of this goal. Additionally, the utmost consequence of this assembly is its high stability, permitting better storage and instantaneous use. The clear change in the color of the electrospun nanofibrous membrane (NFM) could be used for the direct colorimetric visualization of TNT, as a result of which, the color change can be visualized by the naked eye in normal light conditions. Moreover, this nanofibrous hybrid system is relatively trouble-free and does not require any auxiliary alteration which makes it accessible even to an inexperienced person.

The scheme proposed here involves the incorporation of highly red fluorescent BSA-capped gold clusters ${ }^{7}$ (Au.BSA) into the electrospun nanofibrous membrane termed as Au.BSA@PEO-NFM for TNT detection. The essential characterization details of Au.BSA and Au.BSA@PEO-NFM are presented in the ESI. $\dagger$ The randomly oriented defect-free Au.BSA@PEO NFs with relatively uniform diameter of $150 \pm 20 \mathrm{~nm}$ are shown in Fig. 1a, and their photographs under UV light $\left(\lambda_{\text {ext }} 366 \mathrm{~nm}\right)$ and white light are presented in Fig. S1 (ESI $\dagger$ ). The SEM image of PEO NFs and BSA incorporated PEO NFs have also been studied and are illustrated in Fig. S2 (ESI $\dagger$ ) as a reference. The use of the polymeric matrix and the adopted method has allowed us to obtain a uniform dispersion of gold clusters in the NFs while at the same time maintaining the morphology.

The confocal laser scanning microscopy (CLSM) image of the Au.BSA@PEO-NFs excited at $488 \mathrm{~nm}$ shows the characteristic red emission, see Fig. 1b. The distinct outline characteristic of the NFs, especially their length, makes it possible to utilize a single nanofiber (SNF) for sensing. Further, the intensity of the NF emission was measured at various points on the fibre surface, to ensure their exceptional ability, as presented in Fig. S3 (ESI $\dagger$ ). The STEM image and EDS (STEM-EDS) mapping of the Au.BSA@PEO SNF clearly demonstrate the uniform distribution of gold clusters in the NF (see Fig. 1c) and the size was found to be $\sim 2 \mathrm{~nm}$ as shown in Fig. S4 (ESI $\dagger$ ). The enhanced long term stability with their definite morphology have proved to be added 


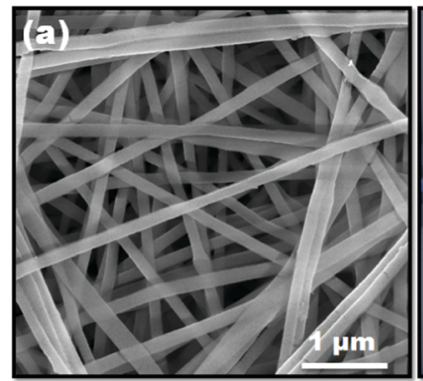

\section{(b)}

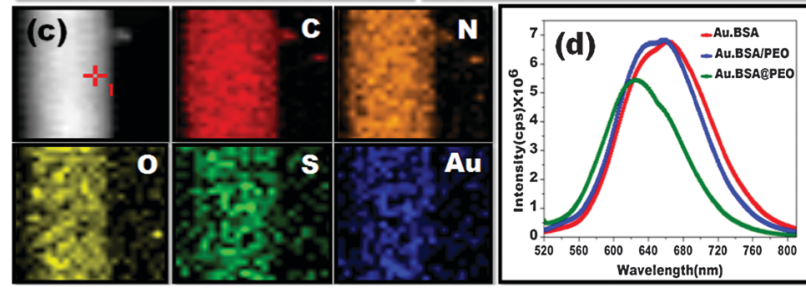

Fig. 1 (a) SEM image of Au.BSA@PEO-NFM; (b) CLSM image of Au.BSA@ PEO-NFM in 3D view, excited at $488 \mathrm{~nm}$; (c) STEM image and EDS mapping of the C, O, N, S and Au elements present in Au.BSA@PEO-NF; (d) emission spectra of Au.BSA, Au.BSA/PEO solution and Au.BSA@PEO-NFM ( $\left.\lambda_{\text {ext }}=500 \mathrm{~nm}\right)$.

advantages of the described system. Thus, the unambiguous character of the NF hybrid system is expected to provide a larger area for binding of the target analyte and, in addition, enhance the performance of the sensor. The emission spectra of Au.BSA, Au.BSA/ PEO solution and Au.BSA@PEO-NFM are depicted in Fig. 1d. The resultant fluorescence emission peak of Au.BSA@PEO-NFM was found to change only slightly, shifting to lower wavelength along with a decreased band width when compared to the solution state. The observed results might be due to the strong confinement and good arrangement of the gold clusters in the NFs which could result from the large specific surface area of the NFs and fast evaporation rate of the solvent during the electrospinning process. ${ }^{9}$ The deconvoluted luminescence spectrum clearly shows the existence of two bands (bands I and II), originating from the Au core and stable $\mathrm{Au}-\mathrm{S}$ bonds respectively as demonstrated by others (see Fig. S5, ESI $\dagger$ ). ${ }^{8}$ There is no distinction in the emission spectra with differences in excitation wavelength as presented in Fig. S6 (ESI $\dagger$ ).

The FTIR and XPS spectra of Au.BSA and Au.BSA@PEONFM are illustrated in Fig. S7 and S8 (ESI $\dagger$ ). The stability of Au.BSA@PEO-NFM was tested for a long period of time in the typical atmosphere and the emission spectra were taken after one and three months (see Fig. S9, ESI $\dagger$ ): there is no discrete decline in the fluorescence intensity highlighting their long term storability and usability. Concluding, the nanofibrous hybrid system is free of self-quenching fluorescence and studies show its stability for a period of three months or more. In addition, no further growth of gold clusters in the PEO NF has been detected, indicating that PEO polymer chains surrounding the gold clusters might possibly hinder the growth of the gold clusters. The sensing performance of Au.BSA@PEO-NFM was tested upon exposure to different concentrations of TNT. It is clearly observed that the fluorescence intensity decreased as a function of increasing concentration of TNT which is demonstrated by CLSM images (Fig. 2a-c). The fluorescence spectral
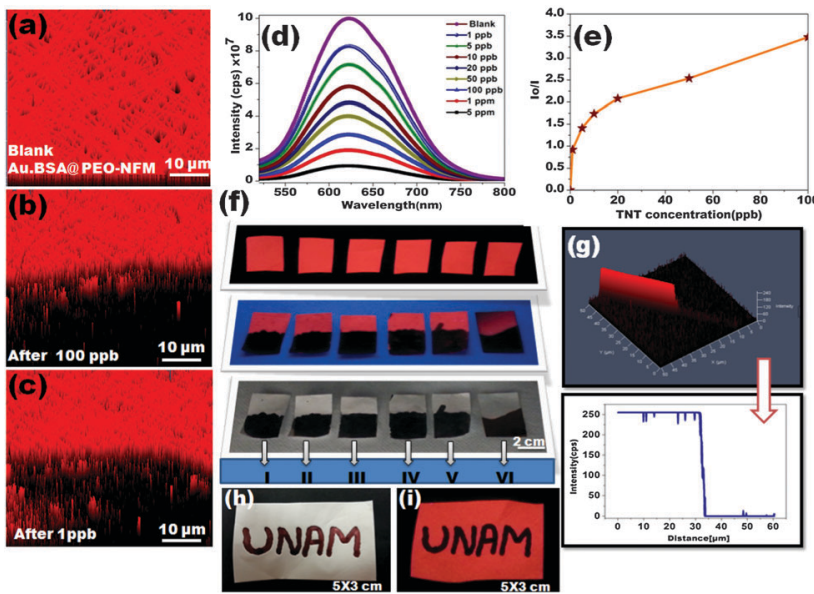

Fig. 2 (a-c) CLSM images of Au.BSA@PEO-NFM on glass slides before (a) and after ( $b$ and $c$ ) exposure to TNT ( $\lambda_{\text {ext }}=488 \mathrm{~nm}, 20 \times$ magnification); (d) fluorescence spectra of Au.BSA@PEO-NFM upon treatment with various concentrations of TNT; (e) relative fluorescence intensity; (f) photograph of the fluorescence quenching of Au.BSACPEO-NFM strips by different concentrations of TNT in contact mode when viewed under UV and white light (I: $1 \mathrm{ppm}$, II: $100 \mathrm{ppb}$, III: $50 \mathrm{ppb}, \mathrm{IV}: 20 \mathrm{ppb}, \mathrm{V}: 10 \mathrm{ppb}, \mathrm{VI}: 1 \mathrm{ppb}$ ); (g) TNT treated Au.BSA@PEO-SNF and the fluorescence intensity profile. (h, i) Hand written patterns on Au.BSA@PEO-NFM using TNT solution as ink.

changes of Au.BSA@PEO-NFM for a wide range of TNT concentrations is illustrated in Fig. 2d. The relative fluorescence intensity $\left(I_{0} / I\right.$, where $I$ and $I_{0}$ are the fluorescence intensity in the presence and absence of TNT, respectively) versus the TNT concentration is presented in Fig. 2e. The visual fluorescence responses of Au.BSA@PEO-NFM at different concentrations of TNT by the contact mode method were tested by placing the NFM in the TNT solution for a second and the results are displayed in Fig. $2 \mathrm{f}$. Immediately, the color of Au.BSA@PEO-NFM changed from white to either deep red or blue depending on the concentration and time in normal light conditions, which clearly illustrates their utility for the onsite instant visualization of TNT. The visible response of the hybrid nanofibrous membrane towards the TNT solution was found to be one part per billion (1 ppb); such a level of sensitivity is lower than the tolerable level of TNT in drinking water, recognized by the US EPA. ${ }^{10,11}$

As observed in the CLSM image, the fluorescence intensity of Au.BSA@PEO-NFM completely decreases upon exposure to $1 \mathrm{ppb}$ TNT, without affecting the NF morphology which has been confirmed by SEM analysis (Fig. S10, ESI $\dagger$ ). Furthermore, a half segment of the isolated single NF was treated with TNT and left to rest to ease visual detection. The intensity profile collected from this SNF is presented in Fig. $2 \mathrm{~g}$. The complete disappearance of luminescence, with no effect on the optical image (see Fig. S11, ESI $\dagger$ ) is observed in the portion treated with TNT whereas there was no decrease in the luminescence in the untreated portion. As expected, the results were more straightforward. Fig. $2 \mathrm{~h}$ and i show a pattern written on Au.BSA@PEO-NFM using TNT solution as ink under normal and UV light, respectively. Another important aspect is the thickness of the nanofibrous mat used for detection, which is also directly interconnected to the expense of the devices. The electrospinning of Au.BSA@PEO NFs 
was carried out for different times (1, 5 and 15 minutes) in order to have different mat thicknesses. The observed results suggested that the sensing performance was not profoundly dependent on the Au.BSA@PEO-NFM thickness, and thin layers of NFs were enough for detection as illustrated in Fig. S12 (ESI $\dagger$ ).

The added flexibility of the hybrid system allows the NFs to be coated on any substrate as per our needs, such as cover slips, silicon wafers, aluminum foil, etc. A similar reduction in quenching efficiency has been observed in Au.BSA@PEO-NFM, which had been stored for prolonged periods of 1 to 3 months as of now, suggesting further prolonging time. Further, the sensing performance in their bulk state has been known to be limited by their cross-linked nature. Moreover, the complete decrease in the fluorescence intensity at one ppb level of TNT in bulk state further prompted us to investigate their sensing performance at even lower concentrations using a SNF, which will further reduce the cost of the devices. As predicted, a SNF could sensitively detect TNT molecules and showed an enhanced detection limit of TNT that reaches sub-ppt levels $(0.1 \mathrm{ppt})$. The gradual decrease in intensity upon increasing the concentration is clearly observed at SNF level as presented in Fig. 3. The decrease in the luminescence intensity occurred consistently throughout the SNF and such excellent uniformity is significant in the development of a reliable method.

The detection of TNT in drinking water has triggered great interest as its intake may cause severe illnesses such as abnormal liver functions, anemia, etc. With this objective, an aqueous solution of TNT was dropped on Au.BSA@PEO-NFM supported by an aluminium substrate. A similar effect was observed for this case too, as illustrated in Fig. S13 (ESI $\dagger$ ). The prepared Au.BSA@PEO-NFM hybrid system has been proved to be well suited for detection of TNT in aqueous media, knowing well that PEO is hydrophilic. A vital feature of the sensor is its selectivity towards the targeted analyte, as a consequence of environmental interference problems encountered in water. Therefore, the response of the sensor towards 2,4-dinitrotoluene (DNT) was tested. No significant quenching effect of the fluorescence was observed and the results confirmed that more apparent difference was seen in the quenching of fluorescence on exposure to TNT vs. DNT at the same concentration. Further, to exclude the interference of metal ions in water, Au.BSA@PEO-NFM was tested against mercury, copper, and zinc ions which are common toxic metal ions present in aqueous media. The results confirmed that the common interfering ions in water have no significant effect on the fluorescence of Au.BSA@PEO-NFM; the fluorescence quenching of TNT, when compared to DNT, was most probably due to the lower electron accepting properties. In addition, it is not able to form an effective Meisenheimer complex with amine groups unlike TNT, resulting in a reduced response. ${ }^{10}$ The fluorescence was slightly quenched when treated with $50 \mathrm{ppm}$ DNT and the result was confirmed by CLSM image as presented in Fig. S15 (ESI $\dagger$ ). Motivated by other groups, we have provided a comparison of our Au.BSA@PEO-NFM hybrid system to Whatman filter paper for detection of TNT. ${ }^{11}$ Highly fluorescent Au.BSA clusters were coated on the filter paper by a dip
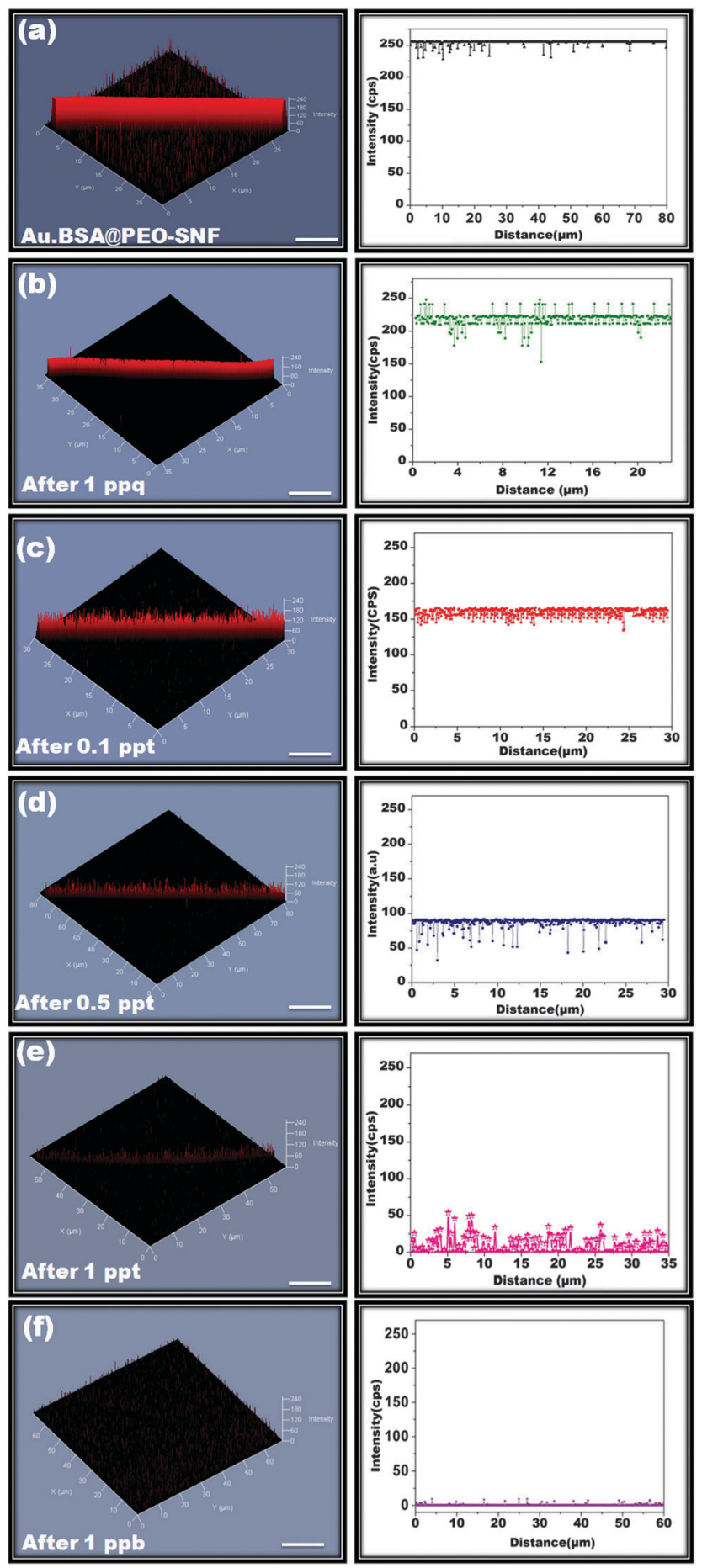

Fig. 3 CLSM images of Au.BSA@PEO-SNF upon exposure to different concentrations of TNT and their intensity profiles collected from the surface of the nanofiber (scale bar $10 \mu \mathrm{m}$ ).

coating method. The basic characterization and their sensing performance of TNT are presented in Fig. S16 (ESI $\dagger$ ). The limit of detection was found to be $1 \mathrm{ppm}$, which is beyond the threshold limit, and the detection definitely was not as prominent as Au.BSA@PEO-NFM. The observed main disadvantages are: (1) the large clusters need to coat a smaller area; (2) the stability is not as good as Au.BSA@PEO-NFM which might be an environmental 
result, and (3) the constrained surface area available to act in response to the analyte. These facts highlight the merits of our nanofibrous hybrid system in terms of improved sensitivity and usage. The superior performance of Au.BSA@PEO-NFM towards TNT should be ascribed to the large energetic surface area for rapid interaction and, more specifically, to the good adsorptive properties of the membrane towards nitroaromatic compounds. ${ }^{12}$ Obviously, the larger surface area of the NF provides a suitable contact space for TNT molecules, and also the entire surface of SNF has been coated with Au.BSA, thereby more active sites are readily available for interaction with TNT molecules with electron deficient effect. At the start, TNT molecules robustly bind to the surface of the NFs through an acid-base interaction between TNT and amino ligands on the NF surface. ${ }^{13}$

Generally, the binding of electron deficient TNT to the amino groups in the BSA was expected to transfer an electron from the amino group to the aromatic ring, which leads to the formation of Meisenheimer complexes between them. ${ }^{14}$ The formation of TNT-amine complexes strongly suppresses the fluorescence emission, which is supported by our experimental results. The amplifying fluorescence quenching can sensitively detect trace TNT, and their specificity enables one to selectively distinguish the different types of nitro compounds. ${ }^{15}$ Moreover, TNT-amine complexes strongly absorb the green part of visible light, and thereby the color of Au.BSA@PEO-NFM changes to deep red. Note that the TNT solution does not absorb any visible part of the light. The results of visible absorption clearly demonstrate the formation of TNT-amine complexes. The experimental concept is in line with other groups also. The superior ability to bind analytes on the surface yields a direct, label-free visual readout.

In summary, we have demonstrated an instant, onsite visual detection of TNT at sub-ppt level using highly fluorescent Au.BSA incorporated onto electrospun PEO NFs. The observed color changes in the presence of TNT make clear that their utility for onsite, instant detection of TNT. The compound exhibited selective recognition of TNT over a wide range of toxic metals in aqueous media. The excellent stability and maintained equivalent activity level after a period of three months were well documented. The exceptional result on single nanofiber detection highlights the unique capability of the hybrid system which has great relevance to human health and greener environment.

A.S. thanks the Scientific \& Technological Research Council of Turkey (TUBITAK) (TUBITAK-BIDEB 2216, Research Fellowship Programme for Foreign Citizens) for a postdoctoral fellowship. A.C. acknowledges TUBITAK-BIDEB for a national PhD study scholarship. T.U. acknowledges partial support of EU FP7-Marie Curie-IRG, NANOWEB (PIRG06-GA-2009-256428) and The Turkish Academy of Sciences - Outstanding Young Scientists Award Program (TUBA-GEBIP). The authors thank M. Guler and Z. Ulger for their technical support for TEM-STEM and CLSM imaging, respectively.

\section{Notes and references}

1 N. Tu and L. Wang, Chem. Commun., 2013, 49, 6319.

2 L. Feng, H. Li, Y. Qu and C. Lu, Chem. Commun., 2012, 48, 4633.

3 J. Yang, S. Aschemeyer, H. P. Martinez and W. C. Trogler, Chem. Commun., 2010, 46, 6804.

4 N. Niamnont, N. Kimpitak, K. Wongravee, P. Rashatasakhon, K. K. Baldridge, J. S. Siegel and M. Sukwattanasinitt, Chem. Commun., 2013, 49, 780.

5 A. Celebioglu and T. Uyar, RSC Adv., 2013, 3, 10197.

6 J. Oberdisse, Soft Matter, 2006, 2, 29.

7 J. Xie, Y. Zheng and J. Y. Ying, J. Am. Chem. Soc., 2009, 131, 888.

8 X. Wen, P. Yu, Y.-R. Toh and J. Tang, J. Phys. Chem. C, 2012, 116, 11830.

9 M. Li, J. Zhang, H. Zhang, Y. Liu, C. Wang, X. Xu, Y. Tang and B. Yang, Adv. Funct. Mater., 2007, 17, 3650.

10 R. Freeman, T. Finder, L. Bahshi, R. Gill and I. Willner, Adv. Mater., 2012, 24, 6416.

11 K. K. Kartha, S. S. Babu, S. Srinivasan and A. Ajayaghosh, J. Am. Chem. Soc., 2012, 134, 4834.

12 Y. Wang, A. La, Y. Ding, Y. Liu and Y. Lei, Adv. Funct. Mater., 2012, 22, 3547 .

13 Y. Engel, R. Elnathan, A. Pevzner, G. Davidi, E. Flaxer and F. Patolsky, Angew. Chem., Int. Ed., 2010, 49, 6830.

14 S. S. R. Dasary, A. K. Singh, D. Senapati, H. Yu and P. C. Ray, J. Am. Chem. Soc., 2009, 131, 13806.

15 Q. Fang, J. Geng, B. Liu, D. Gao, F. Li, Z. Wang, G. Guan and Z. Zhang, Chem. - Eur. J., 2009, 15, 11507. 